\title{
An-Pang Tsai: an exceptional career from quasicrystals to catalysis
}

\author{
M. de Boissieu \\ Univ. Grenoble Alpes, CNRS, Grenoble-INP, F-38000 Grenoble, France, \\ marc.de-boissieu@simap.grenoble-inp.fr
}

An-Pang Tsai, professor at Tohoku University, Sendai, Japan, passed away on May 252019 at the age of 60. He was a pioneer and a leader in the field of quasicrystals and complex intermetallic phases. With him the community has lost one of the brightest scientist in this field. This symposium is dedicated to his memory and illustrates the many different fields he has been contributing in crystallography, metallurgy, material science and solid-state physics and chemistry.

In this presentation we will highlight the many important contributions of the research conducted by An-Pang Tsai going from fundamental and basic research to their applications and the important impact it had on the scientific community. We can quote: the discovery of most of the thermodynamically stable quasicrystals including the stable binary quasicrystals named 'Tsai-type' quasicrystals, the physics allowing to understand which systems is favorable for quasicrystal growth, their atomic structure and physical properties, lattice dynamics, magnetism, mechanical properties. In view of applications he has been one of the first to promote the use of quasicrystals for light alloy reinforcement and more recently he developed a completely new field with new ideas for the development of new catalytic materials.

An-Pang Tsai also had a decisive impact in training the younger generation in the different labs he has ben leading. He always was enthusiast to explore new fields, proposing ambitious targets, but with the long-term perspective, without pressure and with the entire necessary scientific environment. He has coordinated researches in the field of quasicrystals and material sciences in Japan and abroad. In particular he started to organize annual meetings on quasicrystals in Japan in 1996, which continued for more than 20 years andhas organized or initiated number of international conferences.

From very early on in his career, An-Pang Tsai built up an impressive large number of collaborations all across the world, in Japan, Europe, USA, Canada, China and Taiwan. His generosity in sharing his discoveries, his constant curiosity and inspiring ideas have irrigated the quasicrystal community, promoting collaboration rather than competition, leading to major results in almost all aspects of quasicrystal researches and from experiments to theory. He always was available and ready to share his knowledge with senior scientists as well as with young students.

We have lost a great colleague and a good friend, and we will honour his memory by continuing his interdisciplinary research work, keeping his enthusiast and collaborative approach.

[1] de Boissieu M and Ishimasa T (2019) Acta Cryst. B 75763.

Keywords: quasicrystals; catalysis; intermetallics.

Acta Cryst. (2021), A77, C658 